\title{
FLUXO DE POTÊNCIA TRIFÁSICO PROBABILÍSTICO PARA REDES DE DISTRIBUIÇÃO USANDO O MÉTODO DE ESTIMAÇÃO POR PONTOS
}

\author{
Luis Alfonso Gallego* \\ gallegoparejaegmail.com
}

\author{
Mauricio Granada Echeverri* ${ }^{*}$ \\ granadamiieee.org
}

\author{
Antonio Padilha-Feltrin* \\ padilha@dee.feis.unesp.br \\ ${ }^{*}$ Universidade Estadual Paulista - Campus de Ilha Solteira \\ Departamento de Engenharia Elétrica \\ Laboratório de Planejamento de Energia Elétrica, Ilha Solteira, SP, Brasil \\ ${ }^{\dagger}$ Universidad Tecnológica de Pereira \\ Programa de ingeniería eléctrica \\ Grupo de planeamiento eléctrico, Pereira, Risaralda, Colombia
}

\section{ABSTRACT}

Probabilistic three-phase power flow for distribution network using point estimate method

In this paper the point estimation method is applied to solve the probabilistic power flow problem for unbalanced three-phase distribution systems. Through the implementation of this method the probability distribution functions of voltages (magnitude and angle) as well as the active and reactive power flows in the branches of the distribution system are determined. Two different approaches of the point estimation method are presented ( $2 \mathrm{~m}$ and $2 \mathrm{~m}+1$ point schemes). In order to test the proposed methodology, the IEEE 34 and 123 bus test systems are used. The results obtained with both schemes are compared with the ones obtained by a Monte Carlo Simulation (MCS).

KEYWORDS: Probabilistic power flow, distribution system, point estimation method, Monte Carlo simulation, uncertainty.

Artigo submetido em 24/08/2010 (Id.: 1188)

Revisado em 20/12/2010, 03/07/2011, 27/07/2011

Aceito sob recomendação do Editor Associado Prof. Eduardo N. Asada

\section{RESUMO}

Neste artigo é aplicado o método de estimação por pontos para resolver o problema do fluxo de potência trifásico probabilístico para sistemas de distribuição trifásicos desbalanceados. Através da aplicação deste método são determinadas as funções de distribuição de probabilidade das tensões nas barras (magnitude e ângulo) e os fluxos potência ativa e reativa nos ramos da rede. Dois esquemas do método de estimação por pontos são apresentados $(2 \mathrm{~m}$ e $2 \mathrm{~m}+1$ pontos). Para testar os métodos propostos são empregados os sistemas IEEE de 4, 34 e 123 barras. Os resultados obtidos através dos esquemas são comparados aos obtidos por uma simulação de Monte Carlo. Esta análise permite avaliar as potencialidades do método em termos de precisão e tempos de simulação.

PALAVRAS-CHAVE: Fluxo de potência probabilístico, sistemas de distribuição, método de estimação por pontos, simulação de Monte Carlo, Incerteza.

\section{INTRODUÇÃO}

O fluxo de potência (FP) é uma ferramenta fundamental no planejamento e na operação de sistemas elétricos. Através 
da solução do problema podem ser obtidas as condições em regime permanente do sistema, tais como tensões em todas as barras, fluxos de potência ativa e reativa nas linhas, perdas de potência ativa e reativa nas linhas e transformadores, etc.

Tradicionalmente os métodos de solução do FP são denominados determinísticos, devido ao fato que os parâmetros de entrada do problema (demanda nas barras e parâmetros elétricos das linhas) são geralmente modelados de forma determinística (só admitem valores fixos) e não permitem alterações durante o processo de cálculo. Nos sistemas elétricos reais, dados de entrada tais como a demanda e parâmetros elétricos empregados para o cálculo do FP estão sujeitos a incertezas (Borkowska, 1974; Wang and Alvarado, 1992). A incerteza num sistema elétrico pode ocorrer devido a:

- Erros nas medições;

- Imprecisão no cálculo ou prognóstico dos valores de demanda e geração futura;

- Incerteza na distribuição das cargas pelas fases;

- Imprecisão nos cálculos ou medições dos parâmetros dos componentes do sistema.

Várias metodologias foram propostas na literatura especializada para considerar o fenômeno da incerteza nos cálculos do FP para sistemas de transmissão. Estas metodologias são divididas em três grandes grupos: métodos analíticos, métodos de simulação e métodos híbridos (combinação entre analíticos e de simulação).

Métodos analíticos tais como a aritmética de intervalos (Wang and Alvarado, 1992), multilinearização de variáveis (Allan and Leite da Silva, 1981; Leite da Silva and Arienti, 1990), técnicas de convolução (Meliopoulis et al., 1990; Allan et al., 1981), teoria da expansão de Cummulants and Gram-Chanlier (Zhang and Lee, 2004), teoria fuzzy (Miranda et al., 1990; Saraiva et al., 1991), e o método de estimação por pontos (Chun-Lien, 2005; Morales and Pérez-Ruiz, 2007) foram propostos para solucionar o problema do fluxo de potência probabilístico (FPP) nos sistemas de transmissão. Estes métodos são computacionalmente mais efetivos comparados aos métodos de simulação já que em alguns casos precisa de menos iterações para obter uma resposta adequada, mas requerem hipóteses matemáticas.

O método de simulação mais conhecido é o método de Monte Carlo (SMC). Este método foi aplicado com sucesso em muitas áreas da engenharia. A SMC é um procedimento aleatório, que permite determinar probabilisticamente os possíveis valores das variáveis de interesse em determinado instante (Billinton and Li, 1994; Billinton and Allan, 1992). No caso do fluxo de potência probabilístico, a SMC é aplicada para considerar a incerteza natural das cargas dos sistemas elétricos. Neste método as variáveis de interesse são modeladas através das funções de distribuição de probabilidade (FDP) e precisa de rotinas determinísticas. Em (Leite da Silva et al., 1985; Leite da Silva et al., 1984) a SMC foi aplicada para solucionar o problema do FPP em sistemas de transmissão.

Neste artigo é empregado o método de estimação por pontos (MEP) para resolver o problema do FPP para redes de distribuição trifásicas com cargas desbalanceadas. Este método foi aplicado na referência (Chun-Lien, 2005) para um sistema de transmissão de pequeno porte, utilizando o esquema de $2 \mathrm{~m}$ pontos e considerando as incertezas nas cargas e nos parâmetros elétricos das linhas. Uma aplicação do MEP é apresentada na referência (Morales and Pérez-Ruiz, 2007), neste caso, os autores apresentam os esquemas $2 m, 2 m+1$ e $4 m+1$ pontos e são testados os sistemas de transmissão IEEE de 14 e 118 barras e são consideras somente incertezas nas cargas. A proposta deste artigo é formular e verificar o desempenho do MEP para avaliação dos sistemas de distribuição, uma vez que as propostas anteriores fizeram aplicações apenas com sistemas de transmissão. Para testar a aplicabilidade do MEP aos sistemas de distribuição são empregados os esquemas de $2 \mathrm{~m}$ e $2 \mathrm{~m}+1$ pontos nos sistemas trifásicos desbalanceados IEEE de 4, 34 e 123 barras. Uma análise comparativa dos resultados obtidos pelos esquemas apresentados e os obtidos através de uma SMC é realizada com o fim de determinar a eficiência e precisão destes esquemas.

\section{MOTIVAÇÃO}

Os sistemas de distribuição de energia elétrica estão passando por profundas mudanças devido principalmente a políticas de melhoramento da qualidade, segurança e confiabilidade da energia fornecida aos usuários finais. Entre estas políticas encontra-se melhoramento do nível da tensão, diminuição das perdas, correção do fator de potência, diminuição das horas de interrupção do serviço, etc.

No Brasil a situação atual é exatamente esta, sendo que o órgão regulador, a ANEEL, estabeleceu no PRODIST os requerimentos necessários para que os sistemas operem com segurança, eficiência, qualidade e confiabilidade (ANEEL-PRODIST, 2008).

Agora se torna indispensável que a comunidade acadêmica e o setor elétrico possuam ferramentas eficientes para análises dos sistemas elétricos (cálculo do fluxo de potência, estudos de curto circuito, análises de confiabilidade e segurança). Assim como ferramentas estatísticas para determinar as 
probabilidades de ocorrência de eventos no sistema (cálculo do fluxo de potência probabilístico).

\section{MEP PARA RESOLVER O PROBLEMA DO FPP TRIFÁSICO}

Os métodos de MEP foram desenvolvidos para superar dificuldades associadas à falta de um conhecimento completo e perfeito das FDP das variáveis estocásticas. O MEP apresentado neste artigo é um caso particular do método de estimação de pontos proposto em (Hong, 1998), o qual permite calcular os momentos estatísticos das variáveis aleatórias. O método foi aplicado com sucesso para resolver o problema FPP em sistemas de transmissão (Morales et al., 2007, Chun-Lien, 2005), assim como para resolver o problema do fluxo de potência ótimo probabilístico (Verbic et al., 2006).

\subsection{Formulação do problema}

O problema de FPP poder ser formulado matematicamente por dois conjuntos de equações não lineares. Para uma configuração dada da rede, as equações do FP podem ser escritas da seguinte forma (Chun-Lien, 2005):

$$
\begin{aligned}
& Y=G(X) \\
& Z=F(X)
\end{aligned}
$$

sendo:

$$
\begin{array}{lll}
\mathrm{Y} & : \text { Vetor de injeção de potência nas barras; } \\
\mathrm{Z} & : \text { Vetor de variáveis de saída (fluxos de } \\
& \text { potência nas linhas); } \\
\mathrm{X} & : \text { Vetor de variáveis de estado; } \\
G, F: & \text { funções não lineares do fluxo de potência. }
\end{array}
$$

Uma vez que as injeções de potência nas barras são especificadas, o vetor de variáveis de estado pode ser estimado, assim como o vetor das variáveis de saída (fluxos de potência ativa e reativa nas linhas) que é denotado por $Z \mathrm{e}$ pode ser estimado através das equações (1) e (2) da seguinte forma:

$$
\begin{array}{r}
X=G^{-1}(Y) \\
Z=F\left(G^{-1}(Y)\right)
\end{array}
$$

A função $Z$ pode ser reescrita da seguinte forma:

$$
Z=F(W)
$$

sendo $Z$ uma função composta por funções não lineares, que depende das variáveis de estado (X). A solução do FP para o $i$-ésimo termo de $Z$ pode ser escrita da seguinte forma:

$$
Z_{i}=F_{i}\left(p_{1}, p_{2}, \ldots, p_{l}, \ldots, p_{m}\right)
$$

onde $F_{i}$ é uma função não linear e $p_{l}$ é um parâmetro que pode representar a injeção de potência na barra (ativa ou reativa) ou os parâmetros das linhas (resistência ou reatância) do sistema. Uma variação no parâmetro $p_{l}$ causa uma variação na solução do FP. Ao considerar incertezas nos cálculos do FP o parâmetro $p_{l}$ será modificado dependendo da variável aleatória que se considere. Neste artigo o parâmetro $p_{l}$ somente considerara a incerteza na potência injetada nas barras (cargas de potência ativa e reativa).

\subsection{Esquema dois pontos}

Dado que neste trabalho somente será considerado como variáveis incertas as injeções de potência (ativa e reativa) nas barras de carga, então o vetor $W$ é formado pelos elementos $p_{l}$ que denotam o comportamento aleatório da $l$-ésima injeção de potência em um sistema elétrico. O elemento $p_{l}$ possui uma função de distribuição de probabilidade $f_{p l}$. No esquema dois pontos (MEP-2m) são selecionadas duas concentrações ou pontos os quais possuem a máxima probabilidade de ocorrência $\left(p_{l, 1} e p_{l, 2}\right)$ e que podem ser estimados da seguinte forma (Chun-Lien, 2005, Morales et al., 2007):

$$
p_{l, k}=\mu_{p_{l}}+\xi_{l, k} \sigma_{p_{l}}
$$

onde $\mu_{p_{l}}$ e $\sigma_{p_{l}}$ são a média e desvio padrão da FDP de $f_{p l}$ e $\xi_{l, k}$ pode ser estimado da seguinte forma:

$\xi_{l, k}=\frac{\lambda_{l, 3}}{2}+(-1)^{3-k} \times \sqrt{m+\left(\frac{\lambda_{l, 3}}{2}\right)^{2}}, \quad$ com $k=1,2$

onde $\lambda_{l, 3}$ é o coeficiente de assimetria "skewness" de $p_{l}$ e pode ser calculado como:

$$
\lambda_{l, 3}=\frac{E\left[\left(p_{l}-\mu_{p_{l}}\right)^{3}\right]}{\left(\sigma_{p_{l}}\right)^{3}}
$$

sendo $E\left[\left(p_{l}-\mu_{p_{l}}\right)^{3}\right]=\sum_{t=1}^{N}\left(p_{l, t}-\mu_{p l}\right)^{3} \times \operatorname{Prob}\left(p_{l, t}\right), N$ é o número de observações de $p_{l}$ e $\operatorname{Prob}\left(p_{l, t}\right)$ é a probabilidade de cada observação de $p_{l, t}$. 
Uma vez definidos os pontos $p_{l, 1}$ e $p_{l, 2}$, podem ser estimados os valores das funções $Z_{i}(l, 1)$ e $Z_{i}(l, 2)$ executando um FP para cada função.

Os momentos estatísticos $w_{l, k}$ (fatores de peso) são calculados da seguite forma:

$$
w_{l, k}=\frac{1}{m}(-1)^{k} \frac{\xi_{l, 3-k}}{\varsigma_{l}}
$$

onde $m$ é igual ao número de variáveis aleatórias de entrada e $\varsigma_{l}$ é definido como:

$$
\varsigma_{l}=2 \sqrt{m+\left(\frac{\lambda_{l, 3}}{2}\right)^{2}}
$$

Os valores de cada $w_{l, k}$ possuem valores na faixa $[0-1,0]$, e a soma de todos deve ser igual a 1,0 em cada iteração.

\subsection{Esquema três pontos}

O esquema de três pontos é denominado MEP $-2 m+1$ já que, em relação ao esquema $2 m$, precisa de uma avaliação adicional da função $F$ (Morales et al., 2007). No esquema $2 m+1$ são empregadas três concentrações ou pontos para cada variável aleatória, neste caso, uma das concentrações é fixada no valor médio da variável de interesse. Com isto se pode estimar até o quarto momento estatístico da distribuição de probabilidade das variáveis aleatórias.

Os valores de $\xi_{l, k}$ neste caso podem ser estimados da seguinte forma:

$$
\begin{aligned}
& \xi_{l, k}=\frac{\lambda_{l, 3}}{2}+(-1)^{3-k} \times \sqrt{\lambda_{l, 4}-\frac{3}{4} \lambda_{l .3}^{2}}, \quad k=1,2 ; \\
& \xi_{l, 3}=0
\end{aligned}
$$

$\lambda_{l, 3}$ é definido como o coeficiente de assimetria "skewness" de $p_{l}$ e pode ser calculado através da equação (9), e $\lambda_{l, 4}$ é o coeficiente de achatamento (curtose) da variável $p_{l}$ e pode ser estimado por:

$$
\lambda_{l, 4}=\frac{\mu_{4}}{\sigma^{4}}
$$

sendo $\mu_{4}$ o quarto momento estatístico com respeito ao valor médio e ao desvio padrão de $p_{l}$. O coeficiente de achatamento "curtose" de uma variável é o grau de concentração que apresentam os valores dessa variável ao redor da zona central da distribuição de probabilidade.
No caso do esquema $2 m+1$ os momentos estatísticos (pesos) são estimados da seguinte forma:

$$
\begin{aligned}
& w_{l, k}=\frac{(-1)^{3-k}}{\xi_{l, k}\left(\xi_{l, 1}-\xi_{l, 2}\right)} \quad k=1,2 \\
& w_{l, 3}=\frac{1}{m}-\frac{1}{\lambda_{l, 4}-\lambda_{l, 3}^{2}}
\end{aligned}
$$

O esquemas $2 m+1$ é mais preciso que o esquema $2 m$ já que corresponde a uma aproximação de quarta ordem e leva em conta informações dos coeficientes de assimetria e achatamento. Além disso, só requer de uma avaliação adicional da função $F$ (FP adicional) para todo o processo de simulação. Os momentos estatísticos $w_{l, k}$ não são probabilidades, de fato eles podem assumir valores negativos, mas eles são fatores de peso que ao final de cada iteração devem ter soma igual a 1,0.

Nos dois esquemas $(2 m$ e $2 m+1)$, uma vez que as concentrações $\left(p_{l, k}, w_{l, k}\right)$ são obtidas, a função $F$ pode ser avaliada nos pontos $\left(\mu_{p_{1}}, \mu_{p_{2}}, \ldots, p_{l, k}, \ldots, \mu_{p_{m}}\right)$ obtendo-se os valores de $Z(l, k)$. Finalmente, são utilizados os fatores de peso $w_{l, k}$, e os valores de $Z(l, k)$ para estimar o j-ésimo momento das variáveis aleatórias de saída, que podem ser estimados da seguinte forma:

$$
E\left(Z_{i}^{j}\right) \cong \sum_{l=1}^{m} \sum_{k=1}^{3} w_{l, k} \times\left[Z_{i}(l, k)\right]^{j}
$$

O desvio padrão de $Z_{i}$ é calculado como:

$$
\sigma_{Z_{i}}=\sqrt{\operatorname{var}\left(Z_{i}\right)}=\sqrt{E\left(Z_{i}^{2}\right)-\left[E\left(Z_{i}\right)\right]^{2}}
$$

Neste artigo só foram consideradas funções de distribuições normais. No entanto, como é mostrado na referência (Chun-Lien, 2005) o método pode ser aplicado a distribuições não simétricas e com outras funções de distribuição de probabilidade. Considera-se que podem existir múltiplas variáveis aleatórias não correlacionadas entre si. Para o caso particular em que há uma correlação entre as variáveis aleatórias, podem ser empregadas técnicas baseadas em transformações (Hong, 1998).

\subsection{Algoritmo desenvolvido}

O algoritmo proposto para solucionar o problema do FPP através do MEP (esquemas $2 m$ e $2 m+1$ ) é mostrado no fluxograma da figura 1 . 


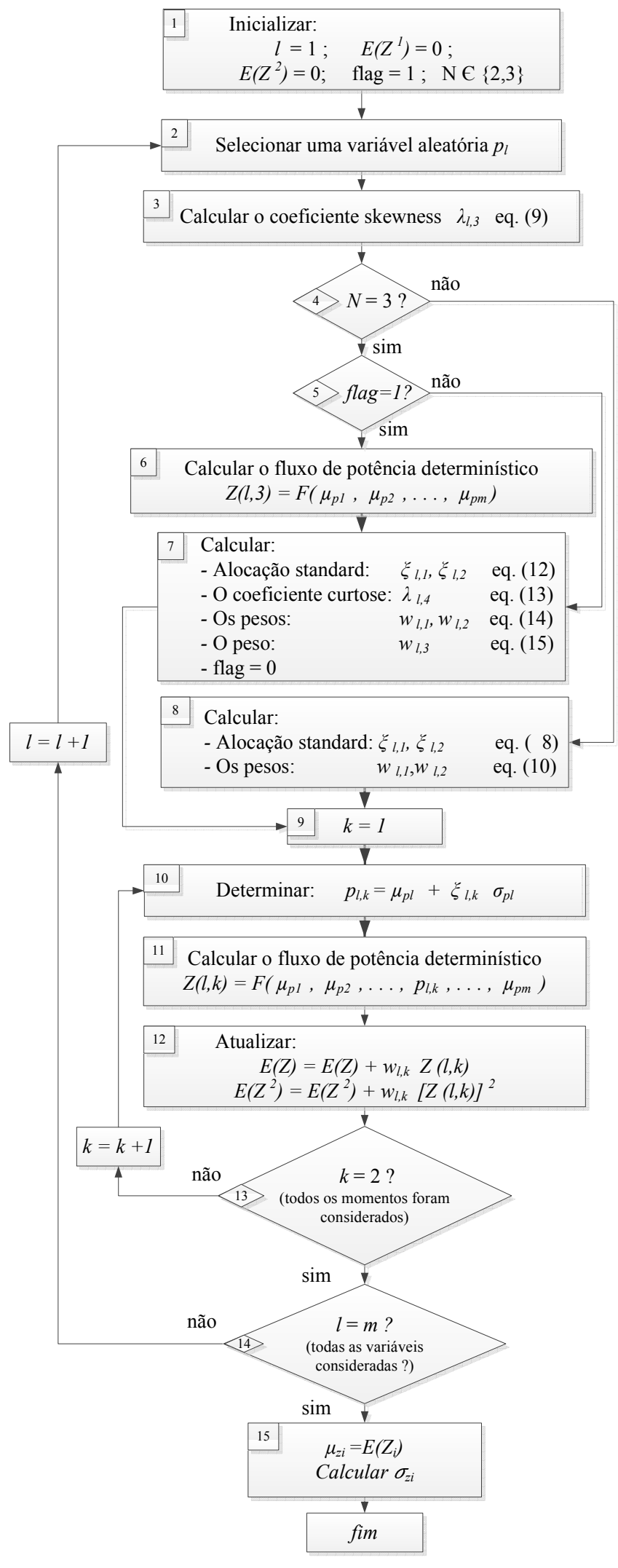

Figura 1: Fluxograma do MEP esquemas $2 m$ e $2 m+1$
No passo 1 são inicializados o contador $l$ e as variáveis flag, $N, E(Z)$ e $E\left(Z^{2}\right)$. No passo 2 é selecionada uma variável do conjunto de variáveis com incertezas. No passo 3 é calculado o coeficiente de skewness da variável aleatória de interesse. O passo 4 é utilizado para identificar qual esquema esta sendo empregado (esquema $2 m$ ou $2 m+1$ ). O passo 5 é utilizado para identificar a primeira iteração do processo de simulação do esquema $2 m+1$, já que nesta iteração se precisa executar um FP determinístico com os valores médios de todas as variáveis aleatórias de interesse (passo 6). No passo 7 são estimados os valores das variáveis $\xi_{l, k}$, os valores dos fatores de peso $w_{l, k}$ e o valor do coeficiente de curtose, a variável flag toma o valor de zero. No passo 8 são estimados os valores das variáveis $\xi_{l, k}$, os valores dos fatores de peso $w_{l, k}$ para o esquema $2 m$ pontos. No passo 9 é inicializado o contador $k$ com um valor de 1 . Os passos 10, 11, $12 \mathrm{e}$ 13 formam um laço, no qual são estimados os dois valores de $p_{l}$ e é executado um FP determinístico para cada valor, também são armazenadas os valores esperados das variáveis de saída $Z$. O passo 14 é utilizado para identificar se todas as variáveis foram selecionadas, caso contrário o algoritmo volta ao passo 2. Finalmente no passo 15 são estimados os valores médios e desvios padrões das variáveis de saída $Z$ (fluxos de potência ativa e reativa nas linhas do sistema) através dos valores contidos nas variáveis $E(z)$ e $E\left(Z^{2}\right)$.

Para solucionar os problemas do FP determinísticos é utilizado o método Backward-Forward Sweep proposto em (Cheng and Shirmohammadi, 1995), mas qualquer método do FP trifásico poder ser empregado. Para um sistema de distribuição com $n$ variáveis aleatórias de entrada o esquema $2 m$ pontos precisa solucionar $2 n$ FP determinísticos. No caso do esquema $2 m+1$ só precisa avaliar um FP adicional considerando os valores médios das demandas iniciais.

Para determinar a eficiência do MEP aplicado ao problema do FPP se pode estimar os erros das variáveis aleatórias de saída $x$ da seguinte forma (Morales et al., 2007):

$$
\begin{aligned}
& \varepsilon_{\mu}^{x}=\left|\frac{\mu_{M C}^{x}-\mu_{M E P}^{x}}{\mu_{M C}^{x}}\right| \times 100[\%] \\
& \varepsilon_{\sigma}^{x}=\left|\frac{\sigma_{M C}^{x}-\sigma_{M E P}^{x}}{\sigma_{M C}^{x}}\right| \times 100[\%]
\end{aligned}
$$

onde $\mu_{M C}^{x}$ e $\sigma_{M C}^{x}$ são a média e o desvio padrão calculados com a SMC, que neste caso são considerados como valores de referência. $\mu_{M E P}^{x}$ e $\sigma_{M E P}^{x}$ são a média e o desvio padrão calculados pelo método de estimação por pontos (esquema $2 \mathrm{~m}$ ou $2 \mathrm{~m}+1$ ). Assim, os erros $\bar{\varepsilon}_{\mu}$ e $\bar{\varepsilon}_{\sigma}$ são definidos como os valores médios de $\varepsilon_{\mu}^{x} \mathrm{e} \varepsilon_{\sigma}^{x}$, respectivamente. 


\section{TESTES E RESULTADOS}

Nesta seção são apresentados os resultados dos testes realizados com os dois esquemas para os sistemas IEEE de 4, 34 e 123 barras. Estes sistemas de distribuição são caracterizados por terem uma configuração trifásica com cargas desequilibradas. Informações dos dados determinísticos podem ser encontradas na referência (Kersting, 1991).

$\mathrm{Na}$ metodologia proposta para solucionar o problema do FPP as cargas do sistema devem ser modeladas através das FDP. Para determinar estas distribuições é preciso contar com valores médios e desvios padrões das cargas. No caso dos sistemas testados são considerados como valores médios os valores nominais de entrada e é considerada uma incerteza máxima de 5\% nas cargas. Assim, por exemplo: se a potência ativa em determinada barra é igual a 15,0 $\mathrm{kW}$, então, esta demanda pode variar $\pm 0,75 \mathrm{~kW}$. Segundo a estatística, quando se considera uma FDP normal existe uma probabilidade de $99,9 \%$ que os dados de uma amostra estejam dentro da faixa: $\mathrm{f}(\mathrm{x})=\mu \pm 3,01 * \sigma$. Através do valor máximo da $\operatorname{FDP}\left(f_{\max }=\mu+3,01 * \sigma\right)$ e com o valor médio se pode determinar o valor do desvio padrão como: $\sigma=\left(f_{\max }\right.$ - $\mu$ ) / 3,01. Assim, o desvio padrão para o exemplo é igual a $\sigma=(15,75-15,0) / 3,01=0,25 \mathrm{~kW}$. Desta forma serão estimados todos os desvios padrões das cargas (potência ativa e reativa) dos sistemas de distribuição a serem testados.

Para analisar a precisão e a eficiência do $M E P$ (esquemas $2 m$ e $2 m+1)$ os resultados obtidos são comparados com os obtidos pelo método de $S M C$ realizando 5000 iterações (5000 fluxos de potência determinísticos executados). Um número alto de simulações garante a convergência da $S M C$. Os valores obtidos da $S M C$ são considerados como valores de referência.

Na tabela 1 é mostrado o número de cargas que possuem os três alimentadores testados. Por exemplo: o alimentador IEEE 34 barras tem 13 cargas monofásicas, 5 bifásicas e 10 trifásicas. Através desta informação se pode determinar o número de variáveis aleatórias empregadas no MEP $(m=13 * 1+5 * 2+10 * 3=53)$. Neste método se considera como variáveis aleatórias tanto a potência ativa como a reativa, assim o número total de variáveis é 106. No esquema $2 \mathrm{~m}$ é preciso rodar 212 fluxos de potência e no esquema $2 \mathrm{~m}+1$ é preciso 213.

\subsection{Resultados obtidos para o sistema IEEE de 4 barras.}

O sistema de 4 barras é um sistema de pequeno porte, que serve para mostrar todos os resultados obtidos pelos métodos apresentados neste artigo. Informações dos dados
Tabela 1: Número de variáveis incertas para os sistemas testados.

\begin{tabular}{|c|c|c|c|c|c|}
\hline \multirow{2}{*}{ Sistema } & \multicolumn{3}{|c|}{ Cargas } & Total & \\
\cline { 2 - 5 } & 1 fase & 2 fases & 3 fases & cargas & $\boldsymbol{m}$ \\
\hline IEEE 4 & - & - & 1 & 3 & 6 \\
\hline IEEE 34 & 13 & 5 & 10 & 53 & 106 \\
\hline IEEE 123 & 80 & - & 5 & 95 & 190 \\
\hline
\end{tabular}

determinísticos podem ser consultados no apêndice A. Os erros médios estimados das tensões por fase (magnitude e ângulo) e dos fluxos de potência ativa e reativa obtidos pelos esquemas $2 m$ e $2 m+1$ são apresentados nas tabelas 2 e 3 .

Tabela 2: Erros médios da magnitude e ângulo da tensão para o alimentador IEEE 4 barras (\%).

\begin{tabular}{|c|c|c|c|c|c|}
\hline \multicolumn{2}{|c|}{} & \multicolumn{2}{|c|}{$\boldsymbol{~}$} & \multicolumn{2}{c|}{$\delta$} \\
\hline Método & Fase & $\bar{\varepsilon}_{\mu}$ & $\bar{\varepsilon}_{\sigma}$ & $\bar{\varepsilon}_{\mu}$ & $\bar{\varepsilon}_{\sigma}$ \\
\hline \multirow{3}{*}{$2 \mathrm{~m}$} & $\mathrm{~A}$ & 0,00003 & 0,54734 & 0,70063 & 1,35781 \\
\cline { 2 - 6 } & $\mathrm{B}$ & 0,00221 & 0,72607 & 0,00053 & 0,65567 \\
\cline { 2 - 6 } & $\mathrm{C}$ & 0,00037 & 0,26291 & 0,00095 & 0,58465 \\
\hline \multirow{3}{*}{$2 \mathrm{~m}+1$} & $\mathrm{~A}$ & 0,00003 & 0,54678 & 0,700063 & 1,35511 \\
\cline { 2 - 6 } & $\mathrm{B}$ & 0,00221 & 0,72549 & 0,00053 & 0,65502 \\
\cline { 2 - 6 } & $\mathrm{C}$ & 0,00037 & 0,26116 & 0,00095 & 0,58416 \\
\hline
\end{tabular}

Tabela 3: Erros médios dos fluxos nas linhas para o alimentador IEEE 4 barras (\%).

\begin{tabular}{|c|c|c|c|c|c|}
\hline \multicolumn{2}{|c|}{} & \multicolumn{2}{|c|}{$\boldsymbol{P}_{i j}$} & \multicolumn{2}{c|}{$\boldsymbol{Q}_{i j}$} \\
\hline Método & Fase & $\bar{\varepsilon}_{\mu}$ & $\bar{\varepsilon}_{\sigma}$ & $\bar{\varepsilon}_{\mu}$ & $\bar{\varepsilon}_{\sigma}$ \\
\hline \multirow{3}{*}{$2 \mathrm{~m}$} & $\mathrm{~A}$ & 0,00630 & 0,58137 & 0,03038 & 0,64665 \\
\cline { 2 - 6 } & $\mathrm{B}$ & 0,03902 & 1,39596 & 0,031887 & 0,14575 \\
\cline { 2 - 6 } & $\mathrm{C}$ & 0,03786 & 0,31236 & 0,01727 & 0,58561 \\
\hline \multirow{3}{*}{$2 \mathrm{~m}+1$} & $\mathrm{~A}$ & 0,00630 & 0,57952 & 0,03038 & 0,64650 \\
\cline { 2 - 6 } & $\mathrm{B}$ & 0,03902 & 1,39437 & 0,03187 & 0,14107 \\
\cline { 2 - 6 } & $\mathrm{C}$ & 0,03786 & 0,31235 & 0,01727 & 0,58798 \\
\hline
\end{tabular}

$\mathrm{Na}$ tabela 4 são apresentados os valores médios e os desvios padrões das tensões nas barras do sistema (colunas 3 a 8), utilizando os esquemas de $2 m$ e $2 m+1$ pontos e pela $S M C$. Pode-se notar desta tabela que os valores médios e desvios padrões das tensões nas barras são muito próximos nos três métodos apresentados. Além disso, fazendo o teste estatístico "qui-quadrado" com um nível de significância de $5 \%$ é possível estabelecer que as informações estatísticas armazenadas no vetor $Z$ se ajustam a uma distribuição normal. No entanto, como é mostrado em (Chun-Lien, 2005) o MEP possui a capacidade de encontrar os momentos estatísticos das variáveis aleatórias com funções de distribuição diferentes à normal. Nesta 
Tabela 4: Valores médios e desvios padrões das tensões e fluxos de potência do sistema IEEE de 4 barras.

\begin{tabular}{|c|c|c|c|c|c|c|c|c|c|c|c|}
\hline \multicolumn{2}{c|}{} & \multicolumn{2}{c|}{ Barra 2 } & \multicolumn{2}{c|}{ Barra 3 } & \multicolumn{2}{c|}{ Barra 4 } & \multicolumn{2}{c|}{$\boldsymbol{P}_{1-2}(\boldsymbol{k W})$} & \multicolumn{2}{c|}{$\boldsymbol{Q}_{1-2}(\boldsymbol{k V A r})$} \\
\hline Método & Fase & $\mu$ & $\sigma$ & $\mu$ & $\sigma$ & $\mu$ & $\sigma$ & $\mu$ & $\sigma$ & $\mu$ & $\sigma$ \\
\hline \multirow{3}{*}{$2 \mathrm{~m}$} & $\mathrm{~A}$ & 0,996 & 0,00019 & 0,989 & 0,00047 & 0,984 & 0,00071 & 1298,337 & 12,802 & 798,009 & 8,243 \\
\cline { 2 - 12 } & $\mathrm{B}$ & 0,986 & 0,00018 & 0,966 & 0,00045 & 0,949 & 0,00067 & 1877,047 & 18,784 & 958,210 & 9,655 \\
\cline { 2 - 12 } & $\mathrm{C}$ & 0,993 & 0,00019 & 0,982 & 0,00046 & 0,973 & 0,00068 & 2389,483 & 24,355 & 943,119 & 9,154 \\
\hline \multirow{3}{*}{$2 \mathrm{~m}+1$} & $\mathrm{~A}$ & 0,996 & 0,00019 & 0,989 & 0,00047 & 0,984 & 0,00071 & 1298,337 & 12,803 & 798,009 & 8,243 \\
\cline { 2 - 11 } & $\mathrm{B}$ & 0,986 & 0,00018 & 0,966 & 0,00045 & 0,949 & 0,00067 & 1877,047 & 18,785 & 958,299 & 9,654 \\
\cline { 2 - 11 } & $\mathrm{C}$ & 0,993 & 0,00019 & 0,982 & 0,00046 & 0,973 & 0,00068 & 2389,483 & 24,355 & 943,119 & 9,154 \\
\hline \multirow{3}{*}{ SMC } & $\mathrm{A}$ & 0,996 & 0,00019 & 0,989 & 0,00047 & 0,984 & 0,00071 & 1298,412 & 12,876 & 797,777 & 8,188 \\
\cline { 2 - 11 } & $\mathrm{B}$ & 0,986 & 0,00018 & 0,966 & 0,00045 & 0,949 & 0,00068 & 1976,300 & 19,054 & 957,973 & 9,640 \\
\cline { 2 - 10 } & $\mathrm{C}$ & 0,993 & 0,00019 & 0,982 & 0,00046 & 0,973 & 0,00068 & 2388,588 & 24,276 & 942,925 & 9,089 \\
\hline
\end{tabular}

mesma tabela também são apresentados o valor médio e desvio padrão da potência ativa $(\mathrm{kW})$ e reativa $(\mathrm{kVAr})$ no início do alimentador para o sistema IEEE de 4 barras (colunas 9 a 12). Um comportamento probabilístico similar é encontrado nos outros dois ramos do sistema, já que nas barras 2 e 3 não tem-se carga. De forma similar que os resultados obtidos para as tensões estimadas nas barras, os valores estimados da média e desvio padrão para o fluxo de potência são muito próximos nos três métodos. Estas diferenças são mais apreciáveis nos desvios padrões dos esquemas $2 m$ e $2 m+1$ com relação aos obtidos pela SMC. Na figura 2 e 3 são apresentadas as FDP e a função de densidade acumulada (FDA) da tensão na fase A para a barra de carga do sistema IEEE de 4 barras.

\subsection{Resultados obtidos para o sistema IEEE de 34 barras.}

Os erros médios estimados das tensões por fase (magnitude e ângulo) e dos fluxos de potência ativa e reativa obtidos pelos esquemas $2 m$ e $2 m+1$ são apresentados nas tabelas 5 e 6. Na tabela 7 são apresentados os valores médios e desvio padrão da potência ativa $(\mathrm{kW})$ e reativa $(\mathrm{kVAr})$ no inicio do alimentador para o sistema IEEE de 34 barras. Nesta tabela, pode-se notar que os resultados obtidos pelo método MEP (esquemas $2 m$ e $2 m+1$ ) são similares aos obtidos pela SMC.

\subsection{Resultados obtidos para o sistema IEEE de 123 barras.}

Os erros médios estimados das tensões por fase (magnitude e ângulo) e dos fluxos de potência ativa e reativa para as três fases do sistema de distribuição IEEE de 123 barras para os esquemas $2 m$ e $2 m+1$ são apresentados nas tabelas 8 e 9.

Na tabela 10 são apresentados os valores médios e desvio padrão da potência ativa $(\mathrm{kW})$ e reativa $(\mathrm{kVAr})$ ao inicio

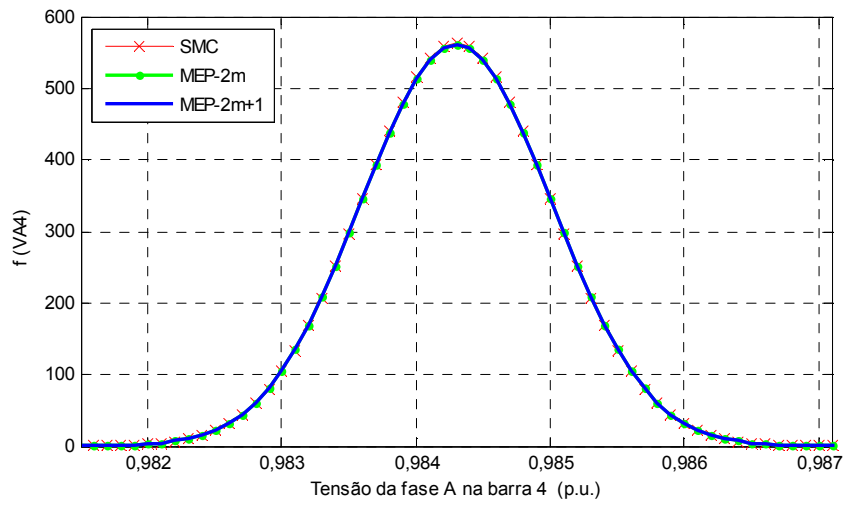

(a) FDP

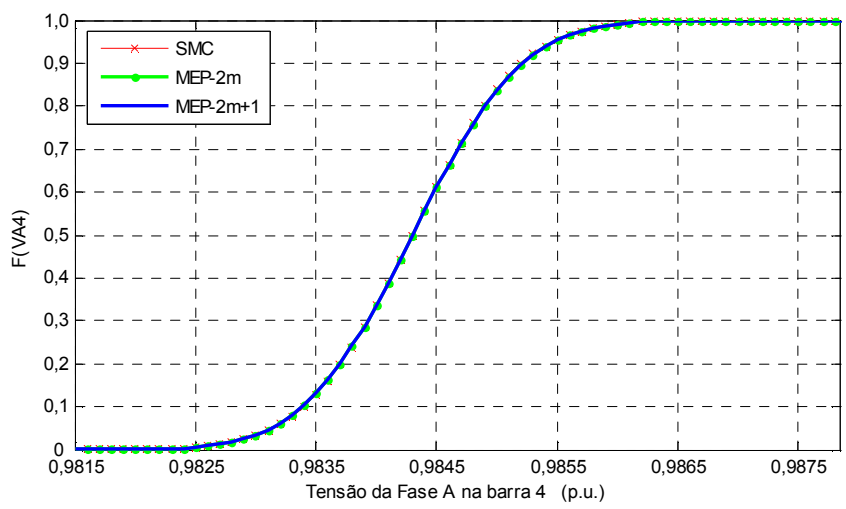

(b) FDA

Figura 2: Comportamento da tensão da fase A na barra 4 do sistema IEEE de 4 barras. 
Tabela 5: Erros médios da magnitude e ângulo da tensão para o alimentador IEEE 34 barras (\%).

\begin{tabular}{|c|c|c|c|c|c|}
\hline \multicolumn{2}{|c|}{} & \multicolumn{2}{|c|}{$\boldsymbol{~}$} & \multicolumn{2}{c|}{$\delta$} \\
\hline Método & Fase & $\bar{\varepsilon}_{\mu}$ & $\bar{\varepsilon}_{\sigma}$ & $\bar{\varepsilon}_{\mu}$ & $\bar{\varepsilon}_{\sigma}$ \\
\hline \multirow{3}{*}{$2 \mathrm{~m}$} & $\mathrm{~A}$ & 0,00385 & 0,21781 & 0,01009 & 0,09155 \\
\cline { 2 - 6 } & $\mathrm{B}$ & 0,00617 & 0,62789 & 0,00056 & 1,33863 \\
\cline { 2 - 6 } & $\mathrm{C}$ & 0,00936 & 0,43937 & 0,00032 & 1,14420 \\
\hline \multirow{3}{*}{$2 \mathrm{~m}+1$} & $\mathrm{~A}$ & 0,00385 & 0,21147 & 0,01012 & 0,08889 \\
\cline { 2 - 6 } & $\mathrm{B}$ & 0,00618 & 0,60961 & 0,00056 & 1,29965 \\
\cline { 2 - 6 } & $\mathrm{C}$ & 0,00936 & 0,42658 & 0,00032 & 1,11088 \\
\hline
\end{tabular}

Tabela 6: Erros médios dos fluxos nas linhas para o alimentador IEEE 34 barras (\%).

\begin{tabular}{|c|c|c|c|c|c|}
\hline \multicolumn{2}{|c|}{} & \multicolumn{2}{c|}{$\boldsymbol{P}_{i j}$} & \multicolumn{2}{c|}{$\boldsymbol{Q}_{i j}$} \\
\hline Método & Fase & $\bar{\varepsilon}_{\mu}$ & $\bar{\varepsilon}_{\sigma}$ & $\bar{\varepsilon}_{\mu}$ & $\bar{\varepsilon}_{\sigma}$ \\
\hline \multirow{3}{*}{$2 \mathrm{~m}$} & $\mathrm{~A}$ & 0,02268 & 0,64988 & 0,10951 & 0,95947 \\
\cline { 2 - 6 } & $\mathrm{B}$ & 0,02573 & 0,59415 & 0,09488 & 0,90086 \\
\cline { 2 - 6 } & $\mathrm{C}$ & 0,03363 & 0,77848 & 0,18109 & 0,82462 \\
\hline \multirow{3}{*}{$2 \mathrm{~m}+1$} & $\mathrm{~A}$ & 0,02267 & 0,64678 & 0,10949 & 0,95602 \\
\cline { 2 - 6 } & $\mathrm{B}$ & 0,02574 & 0,59293 & 0,09488 & 0,90478 \\
\cline { 2 - 6 } & $\mathrm{C}$ & 0,03364 & 0,76376 & 0,18112 & 0,83501 \\
\hline
\end{tabular}

Tabela 7: Resultados dos valores da potência média e desvio padrão no inicio do alimentador IEEE 34 barras.

\begin{tabular}{|c|c|c|c|c|c|}
\hline \multicolumn{2}{|c|}{} & \multicolumn{2}{|c|}{$\boldsymbol{P}_{i j}(\boldsymbol{k W})$} & \multicolumn{2}{c|}{$\boldsymbol{Q}_{i j}(\boldsymbol{k V A r})$} \\
\hline Método & Fase & $\mu$ & $\sigma$ & $\mu$ & $\sigma$ \\
\hline \multirow{3}{*}{$2 \mathrm{~m}$} & $\mathrm{~A}$ & 712,8651 & 3,526 & 163,0950 & 1,7936 \\
\cline { 2 - 6 } & $\mathrm{B}$ & 675,7995 & 3,282 & 138,2349 & 1,6787 \\
\cline { 2 - 6 } & $\mathrm{C}$ & 703,5052 & 3,880 & 157,8016 & 1,9765 \\
\hline \multirow{3}{*}{$2 \mathrm{~m}+1$} & $\mathrm{~A}$ & 712,8651 & 3,525 & 163,0949 & 1,7931 \\
\cline { 2 - 6 } & $\mathrm{B}$ & 675,7996 & 3,281 & 138,2350 & 1,6782 \\
\cline { 2 - 6 } & $\mathrm{C}$ & 703,5052 & 3,878 & 157,8017 & 1,9756 \\
\hline \multirow{3}{*}{ SMC } & $\mathrm{A}$ & 712,5838 & 3,525 & 162,9475 & 1,7894 \\
\cline { 2 - 6 } & $\mathrm{B}$ & 675,6310 & 3,309 & 138,1141 & 1,6855 \\
\cline { 2 - 6 } & $\mathrm{C}$ & 703,2103 & 3,838 & 157,6115 & 1,9906 \\
\hline
\end{tabular}

Tabela 8: Erros médios da magnitude e ângulo da tensão para o alimentador IEEE 123 barras (\%).

\begin{tabular}{|c|c|c|c|c|c|}
\hline \multicolumn{2}{|c|}{} & \multicolumn{2}{|c|}{$\boldsymbol{V}$} & \multicolumn{2}{c|}{$\delta$} \\
\hline Método & Fase & $\bar{\varepsilon}_{\mu}$ & $\bar{\varepsilon}_{\sigma}$ & $\bar{\varepsilon}_{\mu}$ & $\bar{\varepsilon}_{\sigma}$ \\
\hline \multirow{3}{*}{$2 \mathrm{~m}$} & $\mathrm{~A}$ & 0,00261 & 0,50589 & 0,03428 & 1,4838 \\
\cline { 2 - 6 } & $\mathrm{B}$ & 0,00030 & 0,97749 & 0,00035 & 0,4596 \\
\cline { 2 - 6 } & $\mathrm{C}$ & 0,00174 & 0,60954 & 0,00021 & 0,3915 \\
\hline \multirow{3}{*}{$2 \mathrm{~m}+1$} & $\mathrm{~A}$ & 0,00261 & 0,49116 & 0,03428 & 1,4406 \\
\cline { 2 - 6 } & $\mathrm{B}$ & 0,0030 & 0,94902 & 0,00035 & 0,4463 \\
\cline { 2 - 6 } & $\mathrm{C}$ & 0,00174 & 0,59179 & 0,00021 & 0,3801 \\
\hline
\end{tabular}

do alimentador IEEE de 123 barras. Comparando os erros médios dos desvios padrões das tensões dos esquemas $2 m$ e $2 m+1$, observa-se que o esquema $2 m+1$ apresenta valores menores. Isto demonstra que o esquema $2 m+1$ apresenta uma maior precisão. Os erros são menores neste esquema porque é uma aproximação de quarta ordem que leva em conta informações dos coeficientes de assimetria e achatamento da FDP da variável de interesse.

Na tabela 11 são apresentados os tempos computacionais obtidos na aplicação do MEP para os esquemas $2 m, 2 m+1$ e a $S M C$ para os sistemas de distribuição IEEE de 34 e 123 barras. Pode-se notar que os tempos do MEP são inferiores aos da $S M C$. A diferença entre os tempos computacionais dos esquemas $2 m$ e $2 m+1$ é devido à execução do FP adicional. Assim, para o sistema IEEE 34 barras o tempo de processamento é aproximadamente 17 vezes menor que aos obtidos pela $S M C$ e para o sistema IEEE 123 são aproximadamente 10 vezes.

Nas figuras 3a e 3b são apresentadas as FDA dos fluxos de potência ativa e reativa no inicio do sistema de distribuição IEEE de 34 barras. Estas curvas foram obtidas através dos esquemas $2 m, 2 m+1$ e pela SMC. Estas curvas foram obtidas através das informações probabilística contidas na função $Z$, o qual demonstra que além da precisão na média e no desvio padrão o MEP permite obter, o tipo de FDP das variáveis de interesse. Pode-se notar nas duas figuras que existem pequenas diferenças entre os resultados obtidos pelos esquemas apresentados, mas o desempenho é bastante similar. Estas diferenças podem ser reduzidas aumentando o número de pontos estimados, o que leva a um tempo computacional maior. Quando o número de pontos é elevado o tempo computacional pode ser similar ao utilizado pelo método de SMC.

Os algoritmos empregados para solucionar o problema do FPP foram implementados na linguagem $\mathrm{C}++$, em um computador com um processador Intel Core 2 Duo de $3 \mathrm{GHz}$ com 4 GB de RAM. 


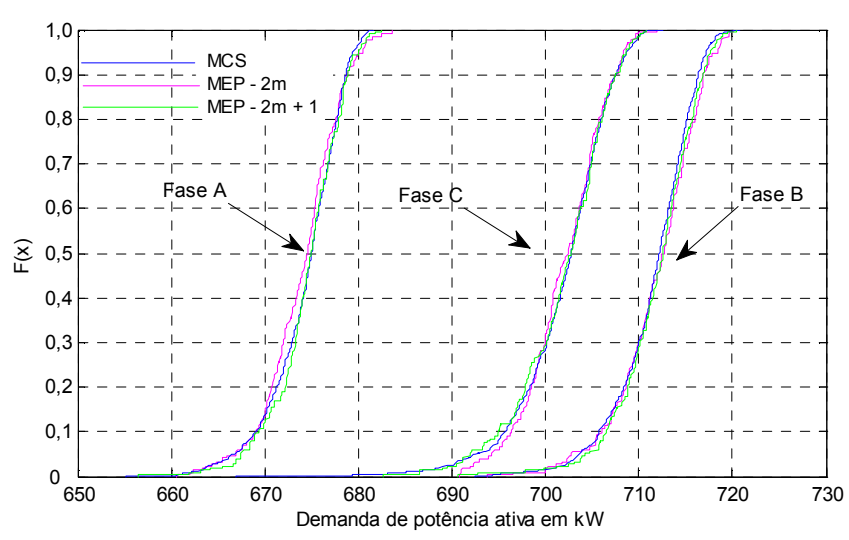

(a) Potência ativa

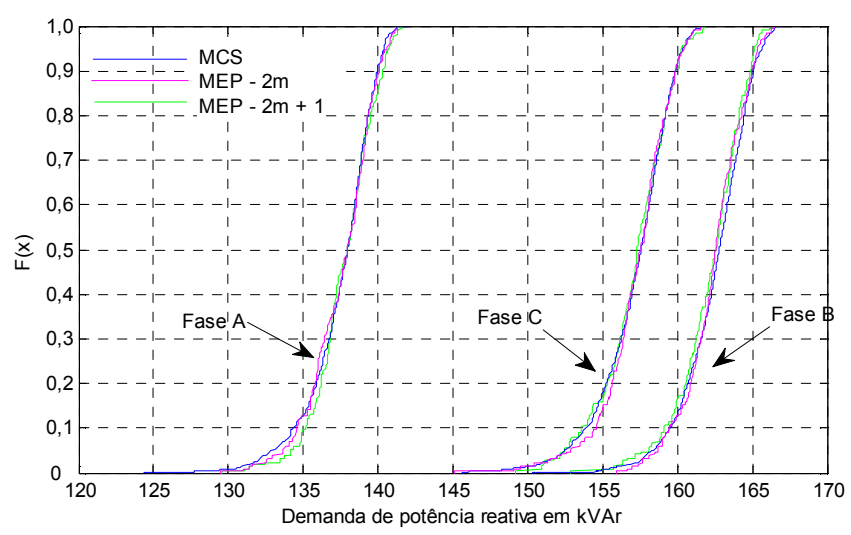

(b) Potência reativa

Figura 3: FDA dos fluxos de potência ao inicio sistema de distribuição IEEE de 34 barras

Tabela 9: Erros médios dos fluxos nas linhas para o alimentador IEEE 123 barras (\%).

\begin{tabular}{|c|c|c|c|c|c|}
\hline \multicolumn{2}{|c|}{} & \multicolumn{2}{c|}{$\boldsymbol{P}_{i j}$} & \multicolumn{2}{c|}{$\boldsymbol{Q}_{i j}$} \\
\hline Método & Fase & $\bar{\varepsilon}_{\mu}$ & $\bar{\varepsilon}_{\sigma}$ & $\bar{\varepsilon}_{\mu}$ & $\bar{\varepsilon}_{\sigma}$ \\
\hline \multirow{3}{*}{$2 \mathrm{~m}$} & $\mathrm{~A}$ & 0,03080 & 0,80897 & 0,03989 & 0,7851 \\
\cline { 2 - 6 } & $\mathrm{B}$ & 0,02626 & 0,66472 & 0,04835 & 0,6643 \\
\cline { 2 - 6 } & $\mathrm{C}$ & 0,02592 & 0,86649 & 0,05035 & 0,8054 \\
\hline \multirow{3}{*}{$2 \mathrm{~m}+1$} & $\mathrm{~A}$ & 0,03080 & 0,80890 & 0,03989 & 0,7814 \\
\cline { 2 - 6 } & $\mathrm{B}$ & 0,02626 & 0,66476 & 0,04835 & 0,6439 \\
\cline { 2 - 6 } & $\mathrm{C}$ & 0,02592 & 0,86640 & 0,05035 & 0,8055 \\
\hline
\end{tabular}

Tabela 10: Resultados dos valores da potência média e desvio padrão no inicio do alimentador IEEE 123 barras.

\begin{tabular}{|c|c|c|c|c|c|}
\hline \multicolumn{2}{|c|}{} & \multicolumn{2}{c|}{$\boldsymbol{P}_{i j}(\boldsymbol{k W})$} & \multicolumn{2}{c|}{$\boldsymbol{Q}_{i j}(\boldsymbol{k V A r})$} \\
\hline Método & Fase & $\mu$ & $\sigma$ & $\mu$ & $\sigma$ \\
\hline \multirow{3}{*}{$2 \mathrm{~m}$} & $\mathrm{~A}$ & 1422,469 & 2,5555 & 621,068 & 1,6008 \\
\cline { 2 - 6 } & $\mathrm{B}$ & 895,768 & 2,0497 & 304,537 & 1,2821 \\
\cline { 2 - 6 } & $\mathrm{C}$ & 1160,275 & 2,3671 & 435,748 & 1,4308 \\
\hline \multirow{3}{*}{$2 \mathrm{~m}+1$} & $\mathrm{~A}$ & 1422,469 & 2,5555 & 621,068 & 1,6008 \\
\cline { 2 - 6 } & $\mathrm{B}$ & 895,768 & 2,0497 & 304,537 & 1,2821 \\
\cline { 2 - 6 } & $\mathrm{C}$ & 1160,275 & 2,3671 & 435,748 & 1,4308 \\
\hline \multirow{3}{*}{ SMC } & $\mathrm{A}$ & 1421,992 & 2,5256 & 620,749 & 1,5909 \\
\cline { 2 - 6 } & $\mathrm{B}$ & 895,513 & 2,0394 & 304,371 & 1,1919 \\
\cline { 2 - 6 } & $\mathrm{C}$ & 1159,955 & 2,3608 & 435,518 & 1,4286 \\
\hline
\end{tabular}

Tabela 11: Tempo (em segundos).

\begin{tabular}{|c|c|c|c|}
\hline Sistema & $\mathbf{2 m}$ & $\mathbf{2 m + 1}$ & SMC \\
\hline IEEE 34 & 0,391 & 0,421 & 6,578 \\
\hline IEEE 123 & 1,125 & 1,172 & 12,344 \\
\hline
\end{tabular}

\section{CONCLUSÕES}

Neste artigo foi apresentada a formulação matemática do MEP para os esquemas dois e três pontos. Este método foi aplicado para solucionar o problema FPP para sistemas de distribuição trifásicos desbalanceados. Através da aplicação do MEP podem-se estimar as funções de distribuição de probabilidade das tensões (magnitude e ângulo) de todas as barras e os fluxos de potência ativa e reativa de todos os ramos do sistema de distribuição.

Uma comparação entre resultados obtidos pelos esquemas $2 m, 2 m+1$ e a SMC para os sistemas testados foi apresentada. Estes resultados mostraram o esquema $2 m+1$ pontos é mais exato que o esquema $2 m$ pontos. Isto se deve ao fato de que o esquema $2 m+1$ tem em conta mais informações das variáveis aleatórias de interesse (coeficiente de assimetria "skewness" e o coeficiente de achatamento "curtose"). Os tempos de execução dos métodos apresentados neste artigo são menores que os obtidos pela SMC.

Os esquemas apresentados permitem obter informações probabilísticas das variáveis de saída que se ajustam adequadamente às informações obtidas através da SMC. Ou seja, o MEP permite obter, além do valor médio e desvio padrão, o tipo de FDP das variáveis de interesse. 
Tabela 12: Dados das cargas dos sistemas IEEE de 4 barras.

\begin{tabular}{|c|c|}
\hline Fase A & $\begin{array}{c}\text { Potência ativa: } 1275 \mathrm{~kW} \\
\text { Fator de potência: } 0,85\end{array}$ \\
\hline Fase B & $\begin{array}{c}\text { Potência ativa: } 1800 \mathrm{~kW} \\
\text { Fator de potência: } 0,90\end{array}$ \\
\hline Fase C & $\begin{array}{c}\text { Potência ativa: } 2375 \mathrm{~kW} \\
\text { Fator de potência: } 0,95\end{array}$ \\
\hline
\end{tabular}

\section{A SISTEMA IEEE 4 BARRAS}

Neste apêndice são apresentados os dados elétricos do sistema IEEE 4 barras. No sistema original que é apresentado nas referências (Kersting et al., 1999) existe um transformador entre as barras 2 e 3 . Para o estudo do FPP realizado neste artigo não foi considerado, assim, foi considerado como um cabo normal com um comprimento de $3000 \mathrm{ft}$.

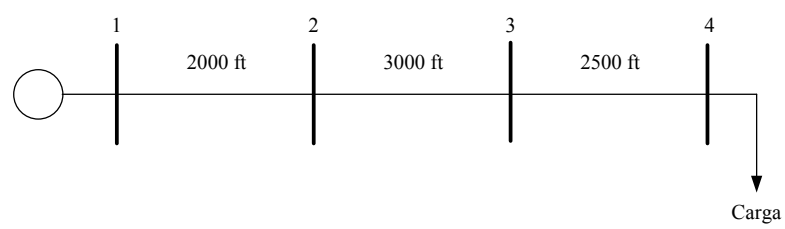

Figura 4: Sistema de distribuição IEEE de 4 barras.

Matriz de impedância em ohm/milha para os cabos do sistema IEEE 4 barras configuração 3 fios.

$Z=\left[\begin{array}{ccc}0,4013+1,4133 j & 0,0953+0,8515 j & 0,0953+0,7266 j \\ 0,0953+0,8515 j & 0,4013+1,4133 j & 0,0953+0,7802 j \\ 0,0953+0,7266 j & 0,0953+0,7802 j & 0,4013+1,4133 j\end{array}\right]$

\section{REFERÊNCIAS}

Allan, R. N. and Leite da Silva, A. M. (1981). Probabilistic load flow using multilinearisations, IEE Proc. C: Generation, Transmission, Distribution 128(5): 280-287.

Allan, R. N., Leite da Silva, A. M. and Burchett, R. C. (1981). Evaluation methods and accuracy in probabilistic load flow solutions, IEEE Trans. Power App. Syst. PAS-100(5): 2539-2546.

ANEEL-PRODIST (2008). Agência Nacional de Energia Elétrica, Procedimentos de Distribuição de Energia Elétrica no Sistema Elétrico Nacional - PRODIST. Disponível em: <http://www.aneel.gov.br/arquivos/PDF>. Acesso em: 31 jul. 2011.
Billinton, R. and Allan, R. N. (1992). Reliability Evaluation of Engineering Systems - Concepts and Techniques, Springer.

Billinton, R. and Li, W. (1994). Reliability assessment of electric power systems using Monte Carlo methods, Springer.

Borkowska, B. (1974). Probabilistic load flow, IEEE Trans. Power App. Syst. PAS-93: 752-755.

Cheng, C. S. and Shirmohammadi, D. (1995). A three phase power flow method for real time distribution system analysis, IEEE Trans. Power Syst. 10(2): 671-679.

Chun-Lien, S. (2005). Probabilistic load-flow computation using point estimate method, IEEE Trans. Power Syst. 20(4): 1843-1851.

Hong, H. P. (1998). An efficient point estimated method for probabilistic analysis, Reliability Engineering and System Safety, Barking 59(3): 261-267.

Kersting, W. H. (1991). Radial distribution test feeders, IEEE Trans. Power Syst. 6(3): 975-985.

Kersting, W. H., Phillips, W. H. and Carr, W. (1999). A new approach to modeling three-phase transformer connections, IEEE Trans. Ind. Appl. 35(1): 169-175.

Leite da Silva, A. M., Allan, R. N. and Arienti, V. L. (1985). Probabilistic load flow considering network outages, IEE Proc. C: Generation, Transmission, Distribution 132(3): 139-145.

Leite da Silva, A. M. and Arienti, V. L. (1990). Probabilistic load flow by a multilinear simulation algorithm, IEE Proc. C: Generation, Transmission, Distribution 137(4): 276-282.

Leite da Silva, A. M., Arienti, V. L. and Allan, R. N. (1984). Probabilistic load flow considering dependence between input nodal powers, IEEE Trans. Power App. Syst. PAS-103(6): 1524-1530.

Meliopoulis, A. P. S., Cokkinides, G. J. and Chao, X. Y. (1990). A new probabilistic power flow analysis method, IEEE Trans. Power Syst. 5: 182-190.

Miranda, V., Matos, M. A. C. C. and Saraiva, J. T. (1990). Fuzzy load flow - new algorithms incorporating uncertain generation and load representation, In Proc. 10th Power Systems Computations Conference, Gras, Austria pp. 621-627.

Morales, J. M. and Pérez-Ruiz, J. (2007). Point estimate schemes to solve the probabilistic power flow, IEEE Trans. Power Syst. 22(4): 1594-1600. 
Saraiva, J. T., Miranda, V. and Matos, M. A. C. C. (1991). Generation and load uncertainties incorporated in load flow studies, In Proceedings of the 6th Mediterranean Electrotechnical Conference 2: 1339-1342.

Verbic, G., Shellenberg, A., Rosehart, W. and Cañizares, C. A. (2006). Probabilistic optimal power flow in electricity markets based on a two-point estimate method, IEEE Trans. Power Syst. 21(4): 1883-1893.

Wang, Z. and Alvarado, F. L. (1992). Interval arithmetic in power flow analysis, IEEE Trans. Power Syst. 7: 1341-1349.

Zhang, P. and Lee, S. T. (2004). Probabilistic load flow computation using the method of combined comulants and gram-charlier expansion, IEEE Trans. Power Syst. 19: $676-682$. 